\title{
Genetic analysis of six SNPs in candidate genes associated with high cross-race risk of development of thoracic aortic aneurysms and dissections in Chinese Han population
}

\author{
Ou LIU, Jian-rong LI, Ming GONG, Ming XU, Jie DU*, Hong-jia ZHANG*
}

The Key Laboratory of Remodeling-related Cardiovascular Diseases, Capital Medical University, Ministry of Education, and Institute of Heart Lung and Blood Vessel Diseases, Beijing Anzhen Hospital Affiliated to the Capital Medical University, Beijing 100029, China

\begin{abstract}
Aim: Genetic susceptibility is an important risk factor for aortic aneurysm and dissection. Recent case-control association studies have identified six single nucleotide polymorphisms (SNPs) associated with abdominal aortic aneurysm (AAA) in a Caucasian population. We aimed to determine whether these loci confer susceptibility to thoracic aortic dissection (TAD) in a Chinese Han population and thus to establish cross-race susceptibility to TAD.

Methods: This study analyzed blood DNA isolated from 206 TAD patients and 180 controls from the ethnic Chinese Han population. Six SNPs - rs819146, rs8003379, rs2853523, rs326118, rs3788205, and rs10757278 - were genotyped using high-throughput matrix-assisted laser desorption ionization-time-of-flight (MALDI-TOF) mass spectrometry.

Results: The A allele frequency for the SNP on 9p21, tagged as rs10757278, was higher in male TAD patients than in male controls $(P=0.017)$. Moreover, with adjustment for traditional cardiovascular risk factors (sex, age, hypertension, dyslipidemia, diabetes, and smoking), the rs 10757278 [odds ratio (OR) 0.63, 95\% confidence interval (Cl) 0.43 to 0.93 ] polymorphism was found to be an independent susceptibility factor for TAD in men.

Conclusion: Our results suggest that a sequence variant on 9p21 is an important susceptibility locus that confers high cross-race risk for development of TAD in Chinese Han population.
\end{abstract}

Keywords: aorta; aneurysm; thoracic aortic dissections; genetic polymorphism; Chinese Han population; males; females

Acta Pharmacologica Sinica (2010) 31: 1376-1380; doi: 10.1038/aps.2010.159; published online 27 Sep 2010

\section{Introduction}

Aneurysms and dissections of the thoracic aorta are the two most catastrophic acute natural events that can befall a human being. Both are major causes of morbidity and mortality in the world $^{[1]}$. Thoracic aortic aneurysms tend to be asymptomatic and usually are not noticed before thoracic aortic dissection (TAD) occurs. It is believed that TAD is a complex trait that is caused by both environmental and genetic factors ${ }^{[2-4]}$. Genetic susceptibility is an important risk factor for TAD. Some genetic syndromes, such as Marfan syndrome and EhlersDanlos syndrome, predispose individuals to $\mathrm{TAD}^{[5,6]}$. Recent studies have also indicated that TAD patients without genetic syndromes tend to have a family history of aortic aneurysm

\footnotetext{
* To whom correspondence should be addressed.

E-mail jdu@bcm.edu (Jie DU); zhanghongjia722@hotmail.com (Hong-jia ZHANG)

Received 2010-05-06 Accepted 2010-08-12
}

disease. However, it remains unclear whether genetic factors influence the formation of TAD in individuals who do not have genetic syndromes.

A single nucleotide polymorphism (SNP) is a common genetic variant that consists of a single DNA base pair change $e^{[7]}$. Several studies have shown that SNPs could explain differences in genetic susceptibility to different diseases. The SNP association study has become a very popular method for identification of genetic factors for complex disease traits ${ }^{[8]}$. Recently, two independent populationbased case-control studies have shown that eight SNPs are associated with abdominal aortic aneurysm in several Caucasian cohorts $^{[9,10]}$. Giusti et al reported seven susceptibility SNPs (such as rs819146, rs8003379, rs2853523, rs326118, and rs3788205) in seven genes involved in methionine metabolism that were associated with abdominal aortic aneurysm formation in an Italian population ${ }^{[9]}$. Helgadottir et al described an association between abdominal aortic aneurysm and a SNP 
on 9p21 in the gene for cyclin-dependent kinase inhibitor in an Icelandic population ${ }^{[10]}$. They found that this SNP, tagged as rs10757278, contributed to the risk of myocardial infarction and intracranial aneurysm. Two other recent independent population-based case-control studies have also shown that the rs10757278 SNP is significantly associated with coronary heart disease and related disorders among Europeans ${ }^{[11,12]}$. The previous studies have found associations between these SNPs and abdominal aortic aneurysm, but only in populations of European descent. Because of the well-known genetic heterogeneity between ethnic populations, validation by genetic case-control studies in non-European populations is essential.

In the present study, we aimed to conduct a case-control association study in a Chinese Han population to test the association between six most-studied SNPs and aortic aneurysm.

\section{Materials and methods}

Study population We enrolled 206 cases that were randomly selected from TAD patients admitted to Beijing Anzhen Hospital affiliated with the Capital Medical University. The criteria for diagnosis of TAD have been previously described ${ }^{[3]}$. In brief, patients were diagnosed by many types of imaging modality, including ultrasound, 2D echocardiography, computed tomography scan, MRI, and angiography. Patients with Marfan syndrome, Ehlers-Danlos syndrome, traumatic aneurysms, or aortic coarctation were excluded from the study. For the control group, 180 volunteers were selected from individuals who were admitted to Beijing Anzhen Hospital for aortic imaging evaluation and were found to have no aortic diseases, such as primary hypertension disease. We chose a subhypertensive age-matched group as the control group because it is known that hypertension is one of the risk factors for TAD. Use of this control group will eliminate the potential contribution of secondary hypertension to TAD. All subjects were unrelated Chinese Han individuals. Clinical information and family histories were collected from medical records and interviews conducted by a genetics counselor. Hypertension was diagnosed as a systolic blood pressure $\geq 140 \mathrm{mmHg}$, a diastolic blood pressure $\geq 90 \mathrm{mmHg}$, or treatment with anti-hypertensive medication. Dyslipidemia was defined as total cholesterol $>6.5 \mathrm{mmol} / \mathrm{L}$ or treatment for elevated blood lipids. Diabetes was defined as a fasting plasma sugar level $>7.8 \mathrm{mmol} / \mathrm{L}$, a glucose level >11.1 mmol/L $2 \mathrm{~h}$ after oral glucose challenge, or ongoing treatment of diabetes. Clinical data, such as fasting insulin levels and glucose levels, were measured routinely for these individuals. Patients and controls gave informed consent to participate in this study, and procedural approval from the Anzhen Hospital Ethics Committee was obtained.

Blood sample collection and genomic DNA extraction Peripheral blood was drawn from a vein into a sterile tube containing ethylenediamine tetraacetic acid (EDTA). Plasma samples were stored at $-80{ }^{\circ} \mathrm{C}$. Genomic DNA was isolated from peripheral blood leukocytes according to the manufacturer's protocol (Genomic DNA kit, Axygen Scientific Inc, California, USA). DNA was stored at $-80^{\circ} \mathrm{C}$ for SNP analysis.

Genotyping We studied six SNPs in or near six candidate genes that were associated with aortic aneurysm according to previous reports ${ }^{[9,10]}$. Five of these genes were suspected to be involved in methionine metabolism. The other was related to a cyclin-dependent kinase inhibitor. Information about these SNPs was acquired from dbSNP NCBI, the ENSEMBL database (http://www.ensembl.org/index.html), and the UCSC Genome Browser website (http:/ / genome.ucsc.edu) (Table 1).

Genotyping was carried out for all SNPs using the MassARRAY platform (Sequenom, San Diego, California USA) according to standard protocols described previously ${ }^{[13]}$. Briefly, probes and primers were designed with Assay Design software (Sequenom) and the genomic DNA samples were amplified with multiplex polymerase chain reaction (PCR). After a multiplex primer extension reaction was performed, terminator nucleotides were added to the diagnostic SNP site. Next, allele-specific extension products of different masses were analyzed in a time-of-flight (MALDI-TOF) SpectroReader mass spectrometer (Sequenom). Finally, the resulting spectra were processed with Typer Analyzer software (Sequenom) and genotype data were generated for the samples. The final genotype call rate of each SNP was greater than $90 \%$ and the total genotyping rate was $99.7 \%$ to ensure the reliability of further statistical analysis.

Statistical analysis Statistical analysis was performed using PLINK 1.06 (http:/ / pngu.mgh.harvard.edu/ purcell/plink/ index.shtml). All genotype distributions of controls in the primary analyses were evaluated for Hardy-Weinberg equilibrium and no evidence of deviations was detected (all exact

Table 1. Description of and information about all investigated single nucleotide polymorphisms (SNPs).

\begin{tabular}{lllll}
\hline \multicolumn{1}{c}{ dbSNPID } & chr position & Gene & Ensemble gene number \\
\hline rs819146 & 20 cen-q13.1 & AHCY & ENSG00000101444 & 5' near gene \\
rs8003379 & $14 q 24$ & MTHFD1 & ENSG00000100714 & intron \\
rs2853523 & $1 q 43$ & MTR & ENSG00000116984 & 3' utr \\
rs326118 & $5 p 15.3-15.2$ & MTRR & ENSG00000124275 & 5' near gene \\
rs3788205 & $21 q 22.3$ & SLC19A1 & ENSG00000035928 & 5' near gene \\
rs10757278 & $9 p 21.3$ & CDKN2BAS & ENSG00000240498 & 3' near gene \\
\hline
\end{tabular}

Chr, chromosome; AHCY, S-adenosylhomocysteine hydrolase; MTHFD1, methylene-tetra hydrofolate dehydrogenase; MTR, methyltetrahydrofolatehomoc ysteine methyltransferase; MTRR, methionine synthase reductase; SLC19A1, solute carrier family 19 (folate transporter), member 1; CDKN2BAS, gene for the cyclin-dependent kinase inhibitor. 
two-sided $P$ values $>0.2)$. Categorical data (presented as frequencies and percentages) were compared between groups using the $\chi^{2}$ test, and continuous data (given as the median and range) were compared using 2 -sample $t$-tests. The Bonferroni correction was applied $(P<0.0083$ based on the 6 SNPs considered in this study). We analyzed the association between each polymorphism and TAD according to different genetic models. Genotype distributions between groups were compared by $x^{2}$ analysis. With adjustment for traditional cardiovascular risk factors (sex, age, hypertension, dyslipidemia, diabetes, and smoking), multiple logistic regression analysis was used to estimate the odds ratio and $95 \%$ confidence intervals for TAD.

\section{Results}

Subjects The demographic and clinical characteristics of both TAD cases and healthy controls are reported in Table 2. Statistically significant gender differences in the occurrence of TAD were observed. Consistent with others' observations ${ }^{[9,14]}$, TAD was substantially more common among men than among women in this study. The clinical data, such as fasting insulin levels and glucose levels, did not differ significantly between cases and controls.

Table 2. Demographic and clinical characteristics of both TAD cases and healthy controls in a Chinese Han population.

\begin{tabular}{lccl}
\hline \multicolumn{1}{c}{ Items } & $\begin{array}{c}\text { Controls } \\
(n=180)\end{array}$ & $\begin{array}{c}\text { TAD patients } \\
(n=206)\end{array}$ & P Value \\
\hline Age & $50.7(16-78)$ & $50.6(21-75)$ & NS \\
Sex (male) $n(\%)$ & $91(50.6)$ & $158(76.7)$ & $<0.0001$ \\
Smoking $n(\%)$ & $105(58.3)$ & $108(52.4)$ & NS \\
Diabetes $n(\%)$ & $27(15)$ & $20(9.7)$ & NS \\
Hypertension $n(\%)$ & $152(84.4)$ & $164(79.6)$ & NS \\
Dyslipidaemia $n(\%)$ & $101(56.1)$ & $110(53.4)$ & NS \\
CAD $n(\%)$ & $16(8.8)$ & $22(10.7)$ & NS \\
\hline
\end{tabular}

TAD, thoracic aortic dissection; CAD, coronary artery disease; NS, not significant. Continuous data were tested using a 2-tailed Student's t-test and categorical data were tested using a Chi-square test (with $d f=1$ ) for the difference between TAD (patient) and control (normal) groups.

\section{Total sample SNP analysis}

As shown in Table 3, allele frequencies and distributions of all SNPs and $\chi^{2}$ analysis according to the dominant model were reported. No evidence for an association between the tested SNPs and the TAD phenotype was detected either at the allele or at the genotype level. In the multiple logistic regression analysis with age, gender, hypertension, diabetes, dyslipidemia, and smoking as the independent variables and TAD as the dependent variable, no significant effect could be observed (data not shown).

SNP analysis of gender-stratified samples To detect possible interactions between gender and SNP genotype status, we stratified the samples according to gender. In the subset of men or women, genotype and allele frequencies did not differ significantly between cases and controls for any of the five genetic variants involved in methionine metabolism (data not shown). However, we found that for the SNP related to cyclin-dependent kinase inhibitor, tagged as rs10757278, a higher proportion of A allele carriers was found among male TAD patients $(57.4 \%)$ than among male controls $(46.1 \%$, $P=0.017)$. Genotype distribution analysis showed statistically significant differences between male patients and male controls, according to the dominant model for rs10757278 (Table 4). Furthermore, we performed logistic regression analysis corrected for the variables age, hypertension, diabetes, dyslipidemia, and smoking in men. The rs 10757278 [odds ratio (OR) $0.63,95 \%$ confidence interval (CI) 0.43 to $0.93, P=0.021$ ] gene polymorphism was an independent genetic susceptibility factor for male TAD patients but not for female TAD patients. However, when we analyzed the 6 candidate SNPs together, we found no significant difference between the groups after the Bonferroni correction.

\section{Discussion}

In view of the disease association found in Caucasian cohorts $^{[9,10]}$, we analyzed six SNPs in a Chinese Han population. We observed an association between the rs10757278 SNP and the occurrence of TAD in Chinese men. Our results suggest that this polymorphism of the gene for a cyclin-dependent kinase inhibitor plays an important role in susceptibility to TAD in a male Chinese Han subpopulation. However, we

Table 3. Genotype distribution and allele frequency of all investigated single nucleotide polymorphisms (SNPs) in thoracic aortic dissection (TAD) patients and control subjects. .

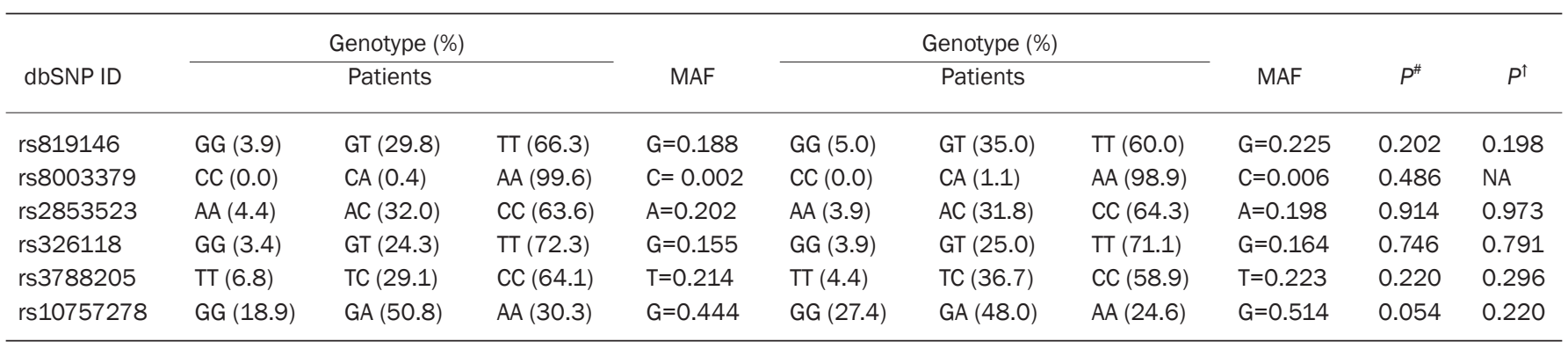

\#, according to the allelic test; $\uparrow$, according to the dominant model; MAF, minor allelic frequency (based on whole sample); NA, not applicable 
Table 4. Differences in the distributions of rs10757278 genotypes and allele frequencies in the gender-stratified subsamples.

\begin{tabular}{|c|c|c|c|c|c|c|c|c|}
\hline \multirow[b]{2}{*}{ dbSNP ID } & \multicolumn{3}{|c|}{ Genotype counts (\%) } & \multicolumn{2}{|c|}{ Allele frequencies } & \multirow[b]{2}{*}{$P^{\#}$} & \multirow[b]{2}{*}{ OR (95\%) } & \multirow[b]{2}{*}{$P^{\uparrow}$} \\
\hline & $\mathrm{G} / \mathrm{G}$ & $\mathrm{G} / \mathrm{A}$ & $\mathrm{A} / \mathrm{A}$ & $\mathrm{G}(\%)$ & $\mathrm{A}(\%)$ & & & \\
\hline TAD cases & 27 (18.1) & $73(49.0)$ & 49 (32.9) & 42.6 & 57.4 & \multirow{2}{*}{0.017} & \multirow{2}{*}{$0.64(0.44-0.92)$} & \multirow{2}{*}{0.032} \\
\hline Controls & $25(27.8)$ & $47(52.2)$ & $18(20.0)$ & 53.9 & 46.1 & & & \\
\hline \multicolumn{9}{|l|}{ Females } \\
\hline Controls & $24(27.0)$ & 39 (43.8) & $26(29.1)$ & 48.9 & 51.1 & 0.861 & $1.05(0.63-1.73)$ & 0.352 \\
\hline
\end{tabular}

\#, according to the allelic test; $\uparrow$, according to the dominant model; OR, estimated odds ratio (for minor allele).

found that the other 5 SNPs that are associated with TAD in a Caucasian population were not associated with the disease in a Chinese population.

Homocysteine, a branch point intermediate of methionine metabolism, has been defined as a new modifiable risk factor for abdominal aortic aneurysm and other vascular diseases ${ }^{[15]}$. Homocysteine may induce overproduction of oxidative radicals ${ }^{[16,17]}$ and activation of matrix metalloproteinase-2 (MMP-2) ${ }^{[18]}$, which are both key mechanisms for aortic aneurysm formation ${ }^{[19,20]}$. Several polymorphisms in genes coding for enzymes involved in methionine metabolism have been suspected to be associated with aortic aneurysm. Recently, a study analyzing the contributions of 56 polymorphisms in 17 genes involved in methionine metabolism on genetic susceptibility to aortic aneurysm was performed. It demonstrated that 7 of these genetic variants are susceptibility factors for aortic aneurysm $^{[9]}$. In the present study, we evaluated the association of 5 major SNPs identified in the above study in a Han Chinese population with aortic dissection. Elevated homocysteine in patients induces oxidative stress, activation of MMP-2 and other pathological stimuli that could contribute to aortic dissection $^{[19]}$. However, no evidence for an association between these 5 polymorphisms and the TAD phenotype was detected either at the allele or at the genotype level. Similarly, casecontrol analysis with gender-stratified samples did not show a significant difference. There are several possible explanations for this discrepancy. First, the association between the tested SNPs involved in methionine metabolism and TAD formation may be ethnicity-specific. Second, environmental factors, such as diet, may contribute to the discrepancy between the studies. For example, homocysteine derived from dietary methionine can be metabolized via two vitamin B pathways ${ }^{[21]}$. Thus, unequal dietary intake of methionine or vitamin B between Western and Chinese populations could contribute to different plasma homocysteine concentrations. Third, the phenotype in our study population was TAD. Compared with other studies on abdominal aortic aneurysm, TAD appeared to be more catastrophic and these dissections tended to rupture easily.

Analysis of the SNP tagged as rs10757278, which is related to an inhibitor of cyclin-dependent kinase, failed to show an association between the sequence variant and TAD in total samples or in the female subset. However, we demonstrated that for this SNP, a higher proportion of A allele carriers was found among male TAD patients (57.4\%) than among male controls $(46.1 \%, P=0.017)$. This SNP is thus a susceptibility factor for TAD in a male Chinese Han subpopulation. The reasons for this sex difference are not clear. It is possible that hormonal factors, increased exposure to important risk factors, genetic susceptibility, or a combination of these variables may contribute to this phenomenon. Our finding is interesting because the SNP rs10757278 has also been found to be associated with cardiovascular diseases $(\mathrm{CAD})^{[11,12,22]}$, diabetes ${ }^{[22]}$, and intracranial aneurysm ${ }^{[23]}$. We have applied Bonferroni's test to our data analyses. The type I error in this type of association study is high, however, we still believe that rs10757278 is a susceptibility factor for thoracic aortic dissection (TAD) in men in the Han Chinese population for several reasons. First, it has been shown that rs10757278 is significantly associated with aortic aneurysm in an Icelandic population. Second, the LD (linkage disequilibrium) block containing this SNP contains two known cyclin-dependent kinase genes, CDKN2A and CDKN2B, as well as a large antisense non-coding RNA, ANRIL. CDKN2A and CDKN2B encode the two cyclindependent kinase inhibitors p16INK4a and p15INK4b, which are known to have critical roles in cell proliferation, aging, senescence and apoptosis $^{[24,25]}$. It is also known that the key mechanism for TAD formation is the proliferation, aging, senescence and apoptosis of smooth muscle cells. In addition, ANRIL, which is $3 \mathrm{~kb}$ from rs10757278, has been shown to be expressed in cells and tissues involved in atherosclerosis ${ }^{[26]}$, which is considered to be a major risk factor for TAD. All of these observations may suggest the potential mechanism of rs10757278 involvement in the pathogenesis of TAD. Together with previous evidence, our study indicates that rs10757278 is a positive SNP for TAD.

In conclusion, a potential association between 6 SNPs and risk of TAD was investigated in a Chinese Han population. We demonstrated that a sequence variant on 9p21, which was related to a cyclin-dependent kinase inhibitor, is an independent susceptibility factor for TAD in a male Han Chinese subpopulation. 


\section{Acknowledgements}

This work was supported by grants from the National Natural Science Foundation Council of China (No 30872536 and No 30888004), the Scientific Research Program of the Beijing Municipal Commission of Education (№ KM200910025020), and the Chinese Ministry of Science and Technology (No 2009CB522205).

\section{Author contribution}

Ou LIU, Jian-rong LI, Jie DU, and Hong-jia ZHANG designed this research; Ou LIU, Jian-rong LI, Ming GONG, and Ming XU performed the research; Ou LIU and Jie DU analyzed the data; and Ou LIU, Jie DU, and Hong-jia ZHANG wrote the paper.

\section{References}

1 Lilienfeld DE, Gunderson PD, Sprafka JM, Vargas C. Epidemiology of aortic aneurysms: I. mortality trends in the United States, 1951 to 1981. Arteriosclerosis 1987; 7: 637-43.

2 Milewicz DM, Guo DC, Tran-Fadulu V, Lafont AL, Papke CL, Inamoto $\mathrm{S}$, et al. Genetic basis of thoracic aortic aneurysms and dissections: focus on smooth muscle cell contractile dysfunction. Annu Rev Genomics Hum Genet 2008; 9: 283-302.

3 Isselbacher EM. Thoracic and Abdominal Aortic Aneurysms. Circulation 2005; 111: 816-28.

4 Guo DC, Papke CL, He R, Milewicz DM. Pathogenesis of thoracic and abdominal aortic aneurysms. Ann N Y Acad Sci 2006; 1085: 33952.

5 Ramirez F, Dietz HC. Marfan syndrome: from molecular pathogenesis to clinical treatment. Curr Opin Genet Dev 2007; 17: 252-8.

6 Gawthrop F, Mould R, Sperritt A, Neale F. Ehlers-Danlos syndrome. BMJ 2007; 335: 448-50.

7 Pettersson FH, Anderson CA, Clarke GM, Barrett JC, Cardon LR, Morris AP, et al. Marker selection for genetic case-control association studies. Nat Protoc 2009; 4: 743-52.

8 Abecasis G, Tam PK, Bustamante CD, Ostrander EA, Scherer SW, Chanock SJ, et al. Human genome variation 2006: emerging views on structural variation and large-scale SNP analysis. Nat Genet 2007; 39: 153-5.

9 Giusti B, Saracini C, Bolli P, Magi A, Sestini I, Sticchi E, et al. Genetic analysis of 56 polymorphisms in 17 genes involved in methionine metabolism in patients with abdominal aortic aneurysm. J Med Genet 2008; 45: 721-30.

10 Helgadottir A, Thorleifsson G, Magnusson KP, Grétarsdottir S, Steinthorsdottir V, Manolescu A, et al. The same sequence variant on 9p21 associates with myocardial infarction, abdominal aortic aneurysm and intracranial aneurysm. Nat Genet 2008; 40: 217-24.

11 Shen GQ, Rao S, Martinelli N, Li L, Olivieri O, Corrocher R, et al. Association between four SNPs on chromosome 9p21 and myocardial infarction is replicated in an Italian population. J Hum Genet 2008; 53: $144-50$.

12 Lemmens R, Abboud S, Robberecht W, Vanhees L, Pandolfo M, Thijs $\mathrm{V}$, et al. Variant on 9p21 strongly associates with coronary heart disease, but lacks association with common stroke. Eur J Hum Genet 2009; 17: 1287-93.

13 Lin YJ, Wan L, Wu JY, Sheu JJ, Lin CW, Lan YC, et al. HLA-E gene polymorphism associated with susceptibility to Kawasaki disease and formation of coronary artery aneurysms. Arthritis Rheum 2009; 60: 604-10.

14 Bown MJ, Lloyd GM, Sandford RM, Thompson JR, London NJ, Samani $\mathrm{NJ}$, et al. The interleukin-10-1082 'A' allele and abdominal aortic aneurysms. J Vasc Surg 2007; 46: 687-93.

15 Brunelli T, Prisco D, Fedi S, Rogolino A, Farsi A, Marcucci R, et al. High prevalence of mild hyperhomocysteinemia in patients with abdominal aortic aneurysm. J Vasc Surg 2000; 32: 531-6.

16 McCully KS. Chemical pathology of homocysteine, II: carcinogenesis and homocysteine thiolactone metabolism. Ann Clin Lab Sci 1994; 24: $27-59$

17 McCully KS. Homocysteine metabolism in scurvy, growth and arteriosclerosis. Nature 1971; 231: 391-2.

18 Bescond A, Augier T, Chareyre C, Charpiot P, Garçon D. Homocysteineinduced elastolysis in arterial media: activation of MMP2. Neth J Med 1998; 58: S56-S57.

19 Griendling KK, FitzGerald GA. Oxidative stress and cardiovascular injury: Part II: animal and human studies. Circulation 2003; 108: 2034-40.

20 Sun J, Sukhova GK, Wolters PJ, Yang M, Kitamoto S, Libby P, et al. Mast cells promote atherosclerosis by releasing proinflammatory cytokines. Nat Med 2007; 13: 719-24.

21 Zhou J, Austin RC. Contributions of hyperhomocysteinemia to atherosclerosis: Causal relationship and potential mechanisms. Biofactors 2009; 35: 120-9.

22 Silander K, Tang H, Myles S, Jakkula E, Timpson NJ, Cavalli-Sforza L, et al. Worldwide patterns of haplotype diversity at 9p21.3, a locus associated with type 2 diabetes and coronary heart disease. Genome Med 2009; 1: 51.

23 Deka R, Koller DL, Lai D, Indugula SR, Sun G, Woo D, et al. The relationship between smoking and replicated sequence variants on chromosomes 8 and 9 with familial intracranial aneurysm. Stroke 2010; 41: 1132-7.

24 Kim WY. Sharpless NE. The regulation of INK4/ARF in cancer and aging. Cell 2006; 127: 265-75.

25 Janzen V, Forkert R, Fleming HE, Saito Y, Waring MT, Dombkowski $\mathrm{DM}$, et al. Stem-cell aging modified by the cyclin-dependent kinase inhibitor p16INK4a. Nature 2006; 443: 421-6.

26 Broadbent HM, Peden JF, Lorkowski S, Goel A, Ongen H, Green F, et al. Susceptibility to coronary artery disease and diabetes is encoded by distinct, tightly linked SNPs in the ANRIL locus on chromosome 9p. Hum Mol Genet 2008; 17: 806-14. 